\title{
Diagonal Earlobe Crease are Associated With Shorter Telomere in Male Japanese Patients With Metabolic Syndrome
}

\author{
A Pilot Study
}

\author{
Yoshihiro Higuchi, MD; Toyoki Maeda, MD; Jing-Zhi Guan, MD; \\ Junichi Oyama, MD; Masahiro Sugano, MD; Naoki Makino, MD
}

\begin{abstract}
Background Diagonal earlobe crease (ELC) have been proposed as a marker of generalized atherosclerosis, so in the present study it was investigated whether individuals with ELC have a shortened telomere, which correlates with an accelerated cell turnover and premature aging, leading to atherosclerosis.

Methods and Results The mean terminal restriction fragment (TRF) was determined by Southern blot hybridization in the peripheral blood cells of 34 male Japanese patients with metabolic syndrome (MetS) who were under 70 years of age with $(n=17)$ and without $(n=17)$ bilateral ELC, and assessed the relationship of ELC to atherosclerotic cardiovascular disease (AVD). The results showed that the TRF was shorter in the MetS patients with ELC in comparison to age- and risk-factor-matched MetS patients without ELC (7.6 $\pm 1.1 \mathrm{kbp}$ vs $8.6 \pm 1.2 \mathrm{kbp}$; $\mathrm{P}<0.05)$. ELC were present in 13 patients in the AVD group $(\mathrm{n}=18)$, but only 4 patients in the non-AVD group $(\mathrm{n}=16)$ had ELC $(72.2 \%$ and $25 \%$ respectively; $\mathrm{P}<0.05)$.

Conclusions These findings suggest that ELC is a useful dermatological indicator of an accelerated aging process, as suggested by excessive telomere loss, and might be a useful indirect marker of high-risk patients. (Circ J 2009; 73: 274-279)
\end{abstract}

Key Words: Aging; Atherosclerosis; Earlobe crease; Metabolic syndrome; Telomere length

$\mathbf{T}$ elomeres are the extreme ends of chromosomal DNA, made up of a large number of tandem repeats of the sequence TTAGGG! Telomeres shorten during the replication of somatic cells, ultimately leading to senescence with progressing biological age? The telomere length in white blood cells (WBCs) reflects the cumulative burden of oxidative stress and inflammation in the circulation during an individual's lifetime ${ }^{3}$ and has been reported to be a useful marker of biological aging of the cardiovascular system.,, 5 Telomere length has been observed to decrease with increases in cardiovascular risk factors such as hypertension, hyperlipidemia, diabetes, obesity and cigarette smoking6 We recently showed that the shortening of the telomeres in peripheral leukocytes was accelerated with advancing age ${ }^{7}$ and it was also observed in patients with sarcoidosis ${ }^{8}$ and Parkinson's disease 9 in the Japanese population.

Since 1973, many studies have demonstrated a correlation between diagonal earlobe crease (ELC) and coronary

(Received March 14, 2008; revised manuscript received August 5, 2008; accepted September 4, 2008; released online December 8, 2008)

Division of Molecular and Clinical Gerontology, Department of Molecular and Cellular Biology, Medical Institute of Bioregulation, Kyushu University, Beppu, Japan

Mailing address: Yoshihiro Higuchi, MD, Division of Molecular and Clinical Gerontology, Department of Molecular and Cellular Biology, Medical Institute of Bioregulation, Kyushu University, 4586 Tsurumihara, Beppu 874-0838, Japan. E-mail: yhiguchi@beppu. kyushu-u.ac.jp

All rights are reserved to the Japanese Circulation Society. For permissions, please e-mail: cj@j-circ.or.jp artery disease (CAD) $!^{10-14}$ Moreover, ELC has also been found to be a marker not only of CAD, but also of generalized atherosclerosis ${ }^{15,16}$ However, the lack of a theoretical foundation for a causal relationship between ELC and atherosclerosis inhibits the clinical value. In previous studies, elastic degeneration of connective tissue and, in a few cases, vascular sclerosis have been observed, leading to speculation regarding premature aging of connective tissue in general as a cause of the correlation between ELC and arteriosclerosis! ${ }^{2}$

To investigate whether patients with ELC have shortened telomeres in comparison to age- and risk-factor-matched subjects without ELC, we studied the telomere length of peripheral blood cells in male Japanese patients with metabolic syndrome (MetS), which is a cluster of glucose intolerance, hypertension and dyslipidemia with visceral fat accumulation, and those with the condition have more prevalent cardiovascular disease or are at greater risk of developing it. ${ }^{17}$ Furthermore, this study is the first to investigate the relationship between bilateral ELC and atherosclerotic cardiovascular diseases (AVD) in patients with MetS. Besides the prevalence of AVD, we examined the brachial-ankle pulse wave velocity (ba-PWV), ankle brachial index (ABI) and intima-media thickness (IMT) of the common carotid artery as physiological markers of arterial stiffness and atherosclerosis 18,19

\section{Methods}

Study Patients

Male MetS patients less than 70 years of age with $(n=17)$ 
and without ELC $(n=17)$ were chosen from patients who were admitted for coronary angiography and were being followed up for treatment of cardiovascular risk factors and for already known AVD. We recruited patients less than 70 years of age because the association between ELC and CAD is ambiguous for patients older than this $!^{3}$

Patients who had evidence of myocardial ischemia or infarction or had a clear medical history of AVD were classified as the AVD group $(n=18)$ in which the patients were all angiographically diagnosed: 14 patients with CAD, 3 with peripheral arterial disease, and 1 with an abdominal aortic aneurysm. Coronary angiography was judged abnormal if there was $>75 \%$ luminal narrowing in the coronary vessels. The patients who underwent coronary angiography were further classified according to the number of diseased arteries showing $>75 \%$ narrowing: 0 -vessel disease (VD, $\mathrm{n}=5), 1-\mathrm{VD}(\mathrm{n}=5)$ and $2-$ or $3-\mathrm{VD}(\geq 2 \mathrm{VD}, \mathrm{n}=9)$, in order to quantify the extent of atherosclerotic involvement of the coronary arteries. In the non-AVD group $(n=16)$, if the resting electrocardiogram (ECG) was normal and the patient did not have any suggestion, sign, or symptoms of AVD, further cardiovascular evaluation was not performed except in patients with diabetes mellitus (DM). In order to rule out silent myocardial ischemia, an exercise ECG was performed in those patients and in the patient who showed a positive result 1-VD CAD was diagnosed by angiography.

DNA samples were taken from peripheral blood samples taken from the patients using 10-ml Vacutainer tubes containing EDTA/heparinized syringes. Written informed consent was given by all patients, and the study was approved by the Conjoint Health Research Ethics Board of Kyushu University.

\section{Grading of ELC}

In each ear, 2 points were given for a deep, clear-cut ELC that extended entirely across the earlobe, 1 point for an ELC that was noted as superficial or did not extend all the way across the earlobe, and 0 for ears in which an ELC was not observed. The ELC score in each case represented the total points for both ears ${ }^{15}$ Bilateral ELC in the present study was defined as a score $\geq 3$.

The appearance of an ELC can change markedly when a subject goes from an upright to a supine position, so each individual was examined while upright 13 The earlobes were digitally photographed for later independent assessment by two of the authors (Y.H., T.M.).

\section{Definition of MetS}

MetS was defined according to the 2005 guidelines of the Japanese Society of Internal Medicine? ${ }^{20}$ According to the Japanese guidelines, which are similar to the International Diabetes Federation criteria,21 subjects with MetS must have abdominal obesity (defined as waist circumference $\geq 85 \mathrm{~cm}$ in men or $\geq 90 \mathrm{~cm}$ in women) plus any 2 of the following 3 factors: (1) dyslipidemia: hypertriglyceridemia (serum triglyceride concentration $\geq 150 \mathrm{mg} / \mathrm{dl}[1.69 \mathrm{mmol} / \mathrm{L}]$ ) and or low high-density lipoprotein-cholesterol (HDL-C serum concentration $\leq 40 \mathrm{mg} / \mathrm{dl}[1.04 \mathrm{mmol} / \mathrm{L}]$ ); (2) hypertension: systolic blood pressure $\geq 130 \mathrm{mmHg}$ and/or diastolic blood pressure $\geq 85 \mathrm{mmHg}$; (3) high fasting glucose: serum glucose concentration $\geq 110 \mathrm{mg} / \mathrm{dl}(6.1 \mathrm{mmol} / \mathrm{L})$.

\section{Measurement of the Terminal Restriction Fragment (TRF) Length}

Telomere detection was performed as previously de-
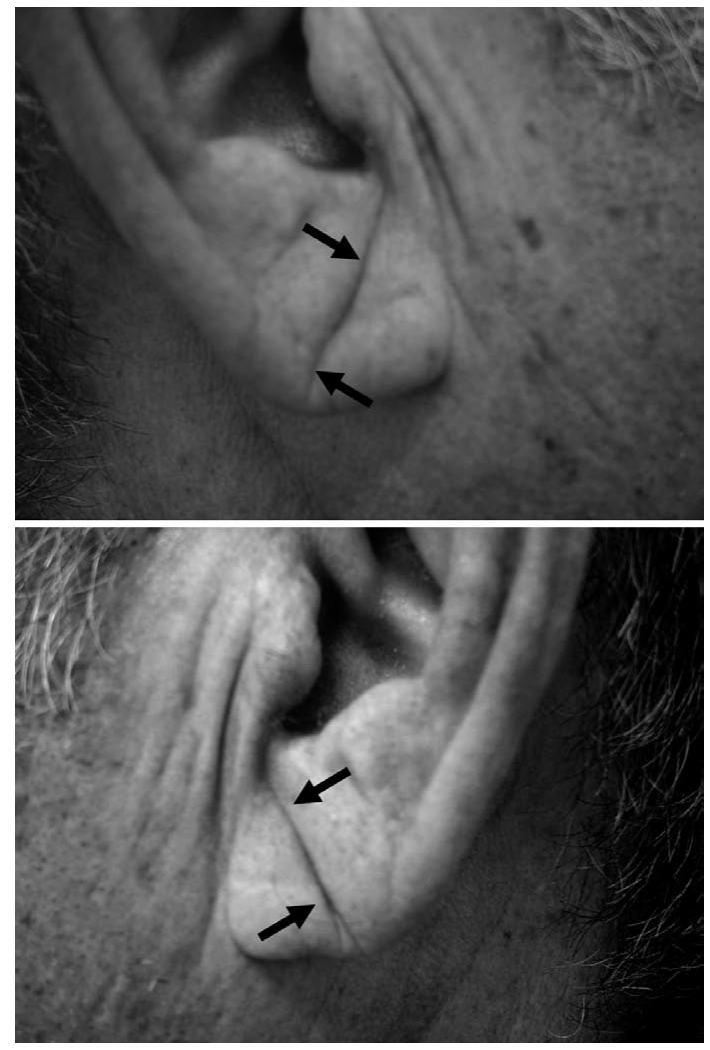

Fig 1. Typical example of the bilateral earlobe crease of a male patient with metabolic syndrome (arrows).

scribed,222,23 Briefly, blood cell DNA was extracted from peripheral blood cells using PureGene DNA Extraction Kits (Gentra Systems, Minneapolis, MN, USA), and the quality was assessed by agarose gel electrophoresis. Aliquots of DNA $(1 \mu \mathrm{g})$ were digested at $37^{\circ} \mathrm{C}$ with $3 \mathrm{U}$ MspI for $2 \mathrm{~h}$. The digests $(20 \mu \mathrm{l})$ were loaded onto a $0.8 \%$ agarose gel (BioRad, Hercules, CA, USA) and resolved by electrophoresis at $100 \mathrm{~V}$ for $30 \mathrm{~min}$. After electrophoresis, the gels were examined by ethidium bromide staining and photographed with a P/N Polaroid film, and then the samples were denatured, neutralized, and transferred by Southern blotting to a positively charged nylon membrane (Roche Diagnostics, Mannheim, Germany). The transfer was done overnight at room temperature using $20 \times \mathrm{SSC}$. The DNA was crosslinked to the nylon membrane using a UV Stratalinker (TM 2400; Stratagene, La Jolla, CA, USA). The blotted DNA fragments were hybridized to a 500-bp-long (TTAGGG)n digoxigenin-labeled probe specific for telomeric repeats. Hybridization was carried out at $42^{\circ} \mathrm{C}$ overnight in a Technehybridizer HB-1D, and the samples were washed as recommended by the manufacturer. The membrane was then incubated with anti-digoxigenin-AP-specific antibody. The telomere probe was visualized by $\mathrm{CSPD}\left(\mathrm{C}_{18} \mathrm{H}_{20} \mathrm{ClO}_{7} \mathrm{PNa}\right)$ (Boehringer Mannheim GmbH, Mannheim, Germany). The membrane was then exposed to Fuji XR film with an intensifying screen. The smears of the autoradiogram were captured on an Image Master 2D Platinum (GE Healthcare UK Ltd, Little Chalfont, UK), and the telomere length was assessed quantitatively. The mean TRF was estimated using the formula: $\sum(\mathrm{ODi}$-background $) / \sum\left(\mathrm{OD}_{\mathrm{i}}-\right.$ background $/ \mathrm{Li}^{24}$ with the mean data from the duplicate runs, where ODi is the chemiluminescent signal and $\mathrm{Li}$ is the length of the TRF 
Table 1 Characteristics of the Study Patients

\begin{tabular}{lcc}
\hline \hline & $E L C$ & Non-ELC \\
\hline$N$ & 17 & 17 \\
Age (years) & $62.5 \pm 4.8$ & $60.1 \pm 4.9$ \\
Body mass index $\left(\mathrm{kg} / \mathrm{m}^{2}\right)$ & $27.0 \pm 3.8$ & $25.5 \pm 2.1$ \\
Abdominal circumference $(\mathrm{cm})$ & $96.5 \pm 9.4$ & $93.1 \pm 5.7$ \\
Systolic blood pressure $(\mathrm{mmHg})$ & $136.1 \pm 14.9$ & $133.8 \pm 17.0$ \\
Diastolic blood pressure $(\mathrm{mmHg})$ & $76.6 \pm 11.3$ & $74.2 \pm 11.1$ \\
Total cholesterol $(\mathrm{mg} / \mathrm{dl})$ & $192.0 \pm 24.1$ & $195.9 \pm 34.6$ \\
Triglycerides $(\mathrm{mg} / \mathrm{dl})$ & $156.2 \pm 90.6$ & $179.5 \pm 81.6$ \\
HDL-cholesterol $(\mathrm{mg} / \mathrm{dl})$ & $47.9 \pm 13.2$ & $42.0 \pm 10.0$ \\
Fasting plasma glucose $(\mathrm{g} / \mathrm{dl})$ & $133.7 \pm 33.4$ & $128.5 \pm 51.5$ \\
Current or ex-smoker, $n(\%)$ & $15(88.2 \%)$ & $15(88.2 \%)$ \\
Hypertension & $15(88.2 \%)$ & $15(88.2 \%)$ \\
Dyslipidemia & $11(64.7 \%)$ & $8(47.1 \%)$ \\
Diabetes melitus & $10(58.8 \%)$ & $6(35.3 \%)$ \\
Diabetes duration $($ years $)$ & $11.6 \pm 7.4$ & $11.3 \pm 7.2$ \\
Medication, $n(\%)$ & & \\
Antihypertensives & $13(76.5 \%)$ & $15(88.2 \%)$ \\
Hypolipidemic drugs & $9(52.9 \%)$ & $7(41.2 \%)$ \\
Antidiabetic drugs & $8(47.1 \%)$ & $6(35.3 \%)$ \\
Statin therapy, $n(\%)$ & $9(52.9 \%)$ & $6(35.3 \%)$ \\
Presence of AVD, $n$ (\%) & $13(76.5 \%) *$ & $5(29.4 \%)$ \\
Carotid IMT (mm) & $0.91 \pm 0.15$ & $0.94 \pm 0.24$ \\
ba-PWV (cm/s) & $1,793 \pm 286$ & $1,613 \pm 337$ \\
Ankle-brachial index & $1.12 \pm 0.08$ & $1.14 \pm 0.10$ \\
\hline
\end{tabular}

Data are mean $\pm S D$ or the number and percentage (\%) of patients. ${ }^{*} P<0.05$ with vs without ELC.

ELC, earlobe crease; HDL, high-density lipoprotein; AVD, atherosclerotic cardiovascular disease; IMT, intimal-medial thickness; ba-PWV, brachialankle pulse wave velocity.

fragment at position i. All analyses were done blinded to the disease status of the individual.

\section{Measurement of Aortic Pulse Wave Velocity (PWV)}

The ba-PWV and ABI were measured using a volumeplethysmograph (form PWV/ABI version-112; Colin Co Ltd, Komaki, Japan), which records PWV, ABI, blood pressure, ECG and heart sounds simultaneously. A detailed description of the measurements, including validity and reproducibility, is provided elsewhere ${ }^{8}$ Briefly, after an overnight fast, the subjects were examined in the supine position, with ECG electrodes placed on both wrists, a microphone for detecting heart sounds placed on the left edge of the sternum, and cuffs wrapped around both of the brachia and ankles. The characteristic points of the waveforms were determined automatically, and the results were then printed out. The mean ba-PWV and ABI values measured on either side of each patient were used for the analysis.

\section{Carotid B-Mode Ultrasound}

Carotid sonography was performed with high-resolution B-mode scanning equipment (ViVid7 GE Healthcare, Milwaukee, WI, USA) with a sector scanner probe that had a bandwidth of $4.0-10.0 \mathrm{MHz}$. The IMT of the common carotid artery was measured in plaque-free segments as the distance from the leading edge of the first echogenic line to that of the second echogenic line. The mean of IMT in the plaque-free segments of both common carotid arteries was used for the analysis 19

\section{Statistical Analysis}

Student's t-test was used to compare continuous variables. The distribution of categorical variables was compared with the chi square $\left(\chi^{2}\right)$ test. Correlations were investigated

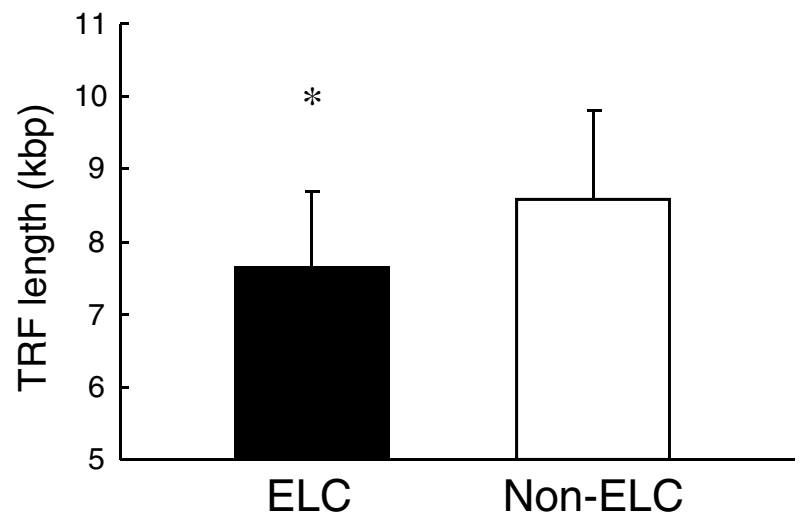

Fig 2. Mean leukocyte terminal restriction fragment (TRF) length is significantly shorter $(\mathrm{P}<0.05)$ in patients with an earlobe crease (ELC: $n=17$ ) than in the patients without a crease (non-ELC: $n=17$ ). The data are presented as the mean \pm standard deviation. ${ }^{*} \mathrm{P}<0.05$ vs non-ELC.

by Spearman's correlation analysis. Either 1-way analysis of variance (ANOVA) followed by Tukey test or the Kruskal-Wallis test was appropriately used to examine the differences among subgroups. The results were presented as the mean \pm standard deviation. The analysis was performed using the SPSS software program for Windows (version 11.5; Chicago, IL, USA). A value of $\mathrm{P}<0.05$ was considered to be significant.

\section{Results}

Table 1 summarizes the population characteristics of the MetS patients with (ELC group, $n=17$ ) and without ELC (non-ELC group, $\mathrm{n}=17$ ). The patients ages ranged similarly in both groups from 51 to 69 years; and the mean age was $62.5 \pm 4.8$ years in the ELC group and $60.1 \pm 4.9$ years in the non-ELC group $(\mathrm{P}>0.05)$. No subjects under the age of 50 were observed to have ELC. The characteristics of the study participants were comparable between both groups with regard to the criteria for the diagnosis of MetS: waist circumference, systolic and diastolic blood pressures, and serum concentrations of glucose, triglyceride and HDL-C. Other risk factors for cardiovascular atherosclerosis, such as hypertension, dyslipidemia, DM, obesity and smoking status, were not significantly different between the groups. The duration of DM did not differ significantly between the groups. There was a similar frequency of using medications for hypertension, dyslipidemia and DM between the groups. Almost all hypolypidemic drugs used were statins, except for 1 patient in the non-ELC group who used bezafibrate.

As shown in Fig 2, the length of the TRF in the peripheral blood cells from the ELC group was $7.6 \pm 1.1 \mathrm{kbp}$ compared with $8.6 \pm 1.2 \mathrm{kbp}$ from the non-ELC group, and the ELC group had a significantly shorter TRF length ( $944 \mathrm{bp}$ less on average) than the non-ELC group $(\mathrm{P}=0.027)$. The telomere attrition rate could not be determined in this study because of the small sample size and narrow range of ages. However, based on our previously reported rate of telomere attrition in the peripheral blood cells of healthy Japanese men (103 bp/year), a difference of $944 \mathrm{bp}$ shorter could account for approximately 9 years of shortened life.

AVD was demonstrated in 13 of 17 (76.5\%) patients in the ELC group (the positive predictive value of ELC) and in 5 of $17(29.4 \%)$ in the non-ELC group (the difference was significant at $\mathrm{P}<0.05)$; therefore, the predictive value 
of the absence of ELC (the negative predictive value) was $70.6 \%$. The sensitivity and specificity of ELC for the diagnosis of AVD were $72.2 \%$ and $75.0 \%$, respectively.

According to the correlation analysis, there was a significant correlation between ELC, TRF length and the presence of AVD. ELC positively correlated with AVD ( $\mathrm{r}=0.359$, $\mathrm{P}=0.037)$. In contrast, ELC was negatively correlated with the TRF length $(\mathrm{r}=-0.377, \mathrm{P}=0.028)$. However, no significant correlation was observed between ELC and the other anthropometric and biological parameters listed in Table 1 (data not shown). In addition to the simple correlation analysis, we computed partial correlation coefficients controlling for the TRF length between ELC and AVD. After controlling for the TRF length, there was no significant correlation between ELC and AVD ( $r=0.299, \mathrm{P}=0.09)$. Our findings suggest that the association between ELC and AVD could be a spurious correlation mediated by the TRF length. A spurious correlation between 2 variables is defined as that which could occur in the absence of any real organic link between the variables. 25

The ELC group tended to show an increase in ba-PWV in comparison with the non-ELC group $(1,793 \pm 286$ vs $1,613 \pm$ $337 \mathrm{~cm} / \mathrm{s}$, respectively), but there was no statistically significant difference between groups $(\mathrm{P}>0.05)$. The $\mathrm{ABI}$ and IMT were comparable between the groups (Table 1).

To investigate the association of TRF length and the extent of coronary artery luminal narrowing, the patients who underwent coronary angiography were classified 0-, 1- and $\geq 2$-VD. As shown in Fig 3A, the TRF length in the patient with 1 - or $\geq 2$-VD was shorter in comparison with the patient with $0-\mathrm{VD}$, and the difference was statistically significant $(\mathrm{P}<0.01)$. However, there was no statistically significant difference of TRF length between $1-\mathrm{VD}$ and $\geq 2$-VD $(\mathrm{P}>0.05)$. To investigate the association of the degree of ELC and the extent of coronary artery luminal narrowing, ELC was graded and defined to range from a score of $0-4$. As shown in Fig 3B, there was a trend toward an increase in ELC score with more severe CAD; however, there was no statistically significant difference among the subgroups.

\section{Discussion}

We demonstrated a shorter telomere length in the MetS patients with ELC than in those without ELC, and a significant correlation between ELC and AVD in MetS patients aged less than 70 years of age. It has been reported that patients with $\mathrm{CAD}$ have a shorter WBC telomere length ${ }^{26}$ and that the shortening of telomeres in the WBC correlates with the degree of cardiovascular damage. The pathogenic significance of telomere shortening in the WBC in the mechanism of atherosclerosis remains uncertain. A possible explanation is that oxidative stress, by enhancing telomere erosion per replication, and inflammation, through increasing WBC turnover, are responsible for the increase in telomere erosion in the $\mathrm{WBC}^{27}$ Both inflammation and oxidative stress are central to the pathology of age-related cardiovascular disorders such as atherosclerosis and arterial stiffness $2^{28}$ Taken together, it is very probable that the high prevalence of AVD in the patients with ELC contributed to telomere shortening in the peripheral blood cells. However, it is unknown whether ELC are linked to telomere shortening through a common genetic factor. Telomere length might be determined genetically because it is highly heritable? 9 Further studies are needed to ascertain whether telomere shortening is the common genetic background of ELC and
A

$*$

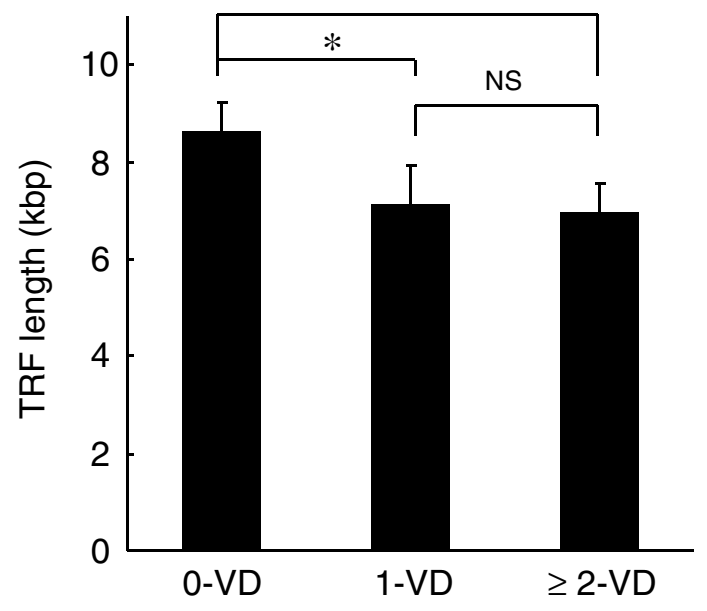

B

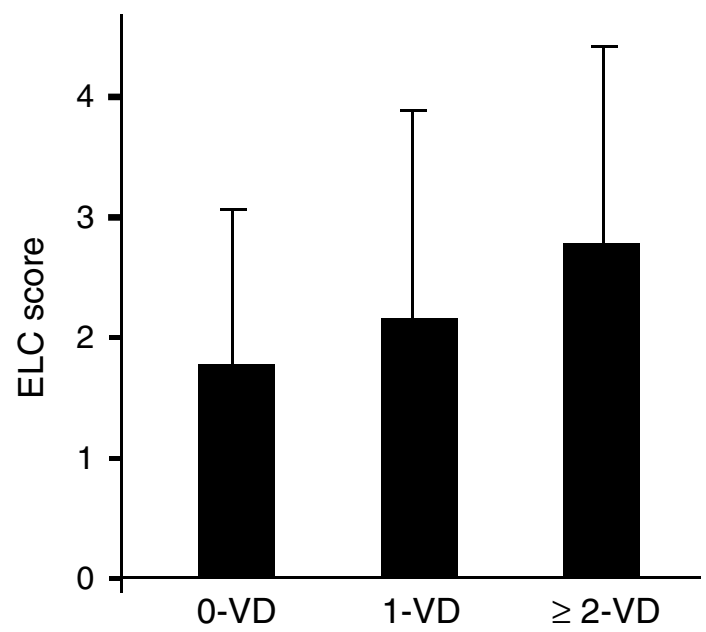

Fig 3. Mean leukocyte terminal restriction fragment (TRF) length (A) and the total score for earlobe crease (ELC) (B) of metabolic syndrome patients with and without significant coronary artery stenosis who were allocated to 1 of 3 groups according to the number of affected coronary vessels: none $(0-\mathrm{VD}, \mathrm{n}=5)$; single-vessel disease $(1-V D, n=5)$; multivessel disease $(\geq 2-V D, n=9)$. The data are presented as the mean \pm standard deviation. $* \mathrm{P}<0.01$ vs 0 -VD; NS, not significant.

generalized atherosclerosis.

In the present study, the mean difference in the TRF length between patients with and without ELC was $944 \mathrm{bp}$. Based on our previously published data? the telomere attrition rate in peripheral blood cells was 103 bp/year in healthy Japanese men. Therefore, the observed difference between patients with and without ELC corresponds to a higher biological age of approximately 9 years. This observation is supported by a prospective observational study by Elliott and Karrison, which demonstrated that ELC are associated with an increased all-cause and cardiac morbidity and mortality ${ }^{30}$ According to the study, the 9-year survival rates for hospitalized patients who had CAD at enrollment were (estimated rate \pm standard error) $29 \pm 9 \%$ (with ELC) and $56 \pm 10 \%$ (without ELC) $?^{30}$

In the present study, the sensitivity, specificity and positive predictive value of ELC for diagnosis of AVD were $72.2 \%, 75.0 \%$ and $76.5 \%$, respectively. In the study by Kuri et al the sensitivity, specificity and positive predictive 
value were $77.0 \%, 84.8 \%$ and $30.3 \%$, respectively, in the preoperative assessment of patients undergoing elective surgery, ranging in age from 50 to 69 years 31 Two other clinical studies of selected populations undergoing coronary angiography have demonstrated that the sensitivity of ELC is $51.3-59.5 \%$, the specificity $81.9-84.8 \%$ and the positive predictive value $89.4-91.1 \%$ !4,32 However, those reports cannot be adequately compared because of differences in race, age, gender, definition of ELC or disease status among the populations studied.

Our data are in good agreement with those of Samani et al who were the first to discover the telomere shortening in leukocytes of patients with $\mathrm{CAD}^{26}$ To our knowledge, the relationship between telomere length and the severity of coronary atherosclerosis has not been reported. In the present study, no significant difference in the TRF length was observed between patients with 1-VD and those with multivessel disease $(\geq 2-\mathrm{VD})$. These findings suggest that the TRF length in WBC may be a poor predictive biomarker for the severity of coronary atherosclerosis.

Prior studies reported that the degree of ELC was related to the extent of coronary atherosclerosis, 13,15 and in the present study, the total ELC score tended to increase with the number of affected coronary vessels; however, the difference among the subgroups did not reach a statistically significant level. These associations or tendencies are not statistically significant because, at least in part, of the small sample size in this study. Further large population study is needed to elucidate the association of the degree of ELC, TRF length and the extent of coronary artery luminal narrowing.

The ba-PWV has been shown to be a marker of the risk of $\mathrm{CAD}^{33}$ and for the extent of coronary atherosclerosis, 34 However, we did not find any statistically significant difference between the ELC and non-ELC groups for ba-PWV. Our results may be partly explained by the findings reported by Koji et al, who demonstrated that ba-PWV values were comparable between groups with and without CAD among subjects with MetS 35 which suggests that the ELC might directly associate with significant atherosclerotic stenosis of the coronary artery independent of its promoting the development of coronary atherosclerosis by increasing arterial stiffness. The present results should be confirmed using a more robust marker of central arterial stiffness, such as the carotid-femoral PWV or augmentation index $3{ }^{3}$ In addition to the ba-PWV, the other atherosclerotic markers (ie, IMT and $\mathrm{ABI}$ ) were comparable between the groups. From these results, we suggest that the presence of ELC, a telomere attrition state, is not associated with carotid atherosclerosis, ba-PWV, or ABI in male Japanese MetS patients.

\section{Study Limitations}

Angiography was only performed in $31.3 \%$ of the nonAVD group. Although the patients in the non-AVD group were free of symptoms and showed normal ECG results at the time they entered the study, there was no definite angiographic proof of the absence of coronary or aortic atherosclerosis. This limitation may have led to an underestimation of the number of patients with AVD. Moreover, in clinical cardiology studies such as the present study, in which the prevalence of CAD is definitely higher because of selection bias, subject selection biases usually occur, as only subjects suspected of having coronary heart disease are investigated, which could have affected the accuracy of the estimated predictive values. Finally, our study population was small, so this study might only be considered a preliminary study.

MetS, a cluster of cardiovascular risk factors, is a common health problem in industrialized countries and its management is a major target for the prevention of AVD. The present study is the first report demonstrating an association between ELC and AVD in MetS patients. Checking ELC in MetS patients could be a useful and cost-free method of identifying high-risk patients in routine clinical practice. We propose that MetS patients with ELC be strongly cautioned to control or reduce other cardiac risk factors in order to prevent future cardiovascular events. In a recent study, Brouilette et al reported that the increased risk of coronary heart disease in patients with shorter telomeres was attenuated by pravastatin treatment ${ }^{36}$ Thus, we speculate that early statin treatment for MetS patients with ELC might prevent AVD. Although this was a small pilot study, the findings could be relevant to the pathogenesis of the association between ELC and atherosclerosis. Further largescale studies are needed to clarify the causative links among telomere attrition, ELC and atherosclerosis.

\section{Acknowledgment}

We acknowledge Mrs Yasuko Ueda for her valuable assistance in extracting DNA.

\section{References}

1. Moyzis RK, Buckingham JM, Cram LS, Dani M, Deaven LL, Jones $\mathrm{MD}$, et al. A highly conserved repetitive DNA sequence, (TTAGGG)n, present at the telomeres of human chromosomes. Proc Natl Acad Sci USA 1988; 85: 6622-6626.

2. Harley $\mathrm{CB}$, Futcher $\mathrm{AB}$, Greider $\mathrm{CW}$. Telomeres shorten during ageing of human fibroblasts. Nature $1990 ; \mathbf{3 4 5}$ : 458-460.

3. Aviv A. Chronology versus biology: Telomeres, essential hypertension, and vascular aging. Hypertension 2002; 40: 229-232.

4. Nakashima H, Ozono R, Suyama C, Sueda T, Kambe M, Oshima T. Telomere attrition in white blood cell correlating with cardiovascular damage. Hypertens Res 2004; 27: 319-325.

5. Benetos A, Gardner JP, Zureik M, Labat C, Xiaobin L, Adamopoulos $\mathrm{C}$, et al. Short telomeres are associated with increased carotid atherosclerosis in hypertensive subjects. Hypertension 2004; 43: 182-185.

6. Valdes AM, Andrew T, Gardner JP, Kimura M, Oelsner E, Cherkas LF, et al. Obesity, cigarette smoking, and telomere length in women. Lancet 2005; 366: 662-664.

7. Guan JZ, Maeda T, Sugano M, Oyama J, Higuchi Y, Makino N. Change in the telomere length distribution with age in the Japanese population. Mol Cell Biochem 2007; 304: 353-360.

8. Guan JZ, Maeda T, Sugano M, Oyama J, Higuchi Y, Suzuki T, et al. An analysis of telomere length in sarcoidosis. J Gerontol A Biol Sci Med Sci 2007; 62: 1199-1203.

9. Guan JZ, Maeda T, Sugano M, Oyama J, Higuchi Y, Suzuki T, et al. A percentage analysis of the telomere length in Parkinson's disease patients. J Gerontol A Biol Sci Med Sci 2008; 63: 467-473.

10. Frank ST. Aural sign of coronary-artery disease. N Engl J Med 1973; 289: $327-328$.

11. Lichstein E, Chadda KD, Naik D, Gupta PK. Diagonal ear-lobe crease: Prevalence and implications as a coronary risk factor. $N$ Engl J Med 1974; 290: 615-616.

12. Shoenfeld Y, Mor R, Weinberger A, Avidor I, Pinkhas J. Diagonal ear lobe crease and coronary risk factors. J Am Geriatr Soc 1980; 28: $184-187$.

13. Tranchesi Junior B, Barbosa V, de Albuquerque CP, Caramelli B, Gebara O, dos Santos Filho RD, et al. Diagonal earlobe crease as a marker of the presence and extent of coronary atherosclerosis. Am J Cardiol 1992; 70: 1417-1420.

14. Evrengul H, Dursunoglu D, Kaftan A, Zoghi M, Tanriverdi H, Zungur $\mathrm{M}$, et al. Bilateral diagonal earlobe crease and coronary artery disease: A significant association. Dermatology 2004; 209: 271-275.

15. Ishii T, Asuwa N, Masuda S, Ishikawa $\mathrm{Y}$, Shimada K, Takemoto $\mathrm{S}$. Earlobe crease and atherosclerosis: An autopsy study. J Am Geriatr Soc 1990; 38: 871-876.

16. Edston E. The earlobe crease, coronary artery disease, and sudden cardiac death: An autopsy study of 520 individuals. Am J Forensic Med Pathol 2006; 27: 129-133. 
17. Grundy SM, Cleeman JI, Daniels SR, Donato KA, Eckel RH, Franklin BA, et al. Diagnosis and management of the metabolic syndrome: An American Heart Association/National Heart, Lung, and Blood Institute Scientific Statement. Executive summary. Cardiol Rev 2005; 13: $322-327$.

18. Yamashina A, Tomiyama H, Takeda K, Tsuda H, Arai T, Hirose K, et al. Validity, reproducibility, and clinical significance of noninvasive brachial-ankle pulse wave velocity measurement. Hypertens Res 2002; 25: 359-364.

19. Taniguchi A, Fukushima M, Kuroe A, Sakaguchi K, Hashimoto H, Yoshioka I, et al. Metabolic syndrome, insulin resistance, and atherosclerosis in Japanese type 2 diabetic patients. Metabolism 2007; 56: $1099-1103$

20. The Committee for the Diagnostic Criteria of Metabolic Syndrome. The definition and diagnostic criteria of metabolic syndrome. $J$ Jpn Soc Int Med 2005; 94: 794-809 (in Japanese, translated by the authors).

21. Alberti KG, Zimmet P, Shaw J. The metabolic syndrome: A new worldwide definition. Lancet 2005; 366: 1059-1062.

22. Vaziri H, Dragowska W, Allsopp RC, Thomas TE, Harley CB, Lansdorp PM. Evidence for a mitotic clock in human hematopoietic stem cells: Loss of telomeric DNA with age. Proc Natl Acad Sci USA 1994; 91: 9857-9860.

23. Cherif H, Tarry JL, Ozanne SE, Hales CN. Ageing and telomeres: A study into organ- and gender-specific telomere shortening. Nucleic Acids Res 2003; 31: 1576-1583.

24. de Lange T, Shiue L, Myers RM, Cox DR, Naylor SL, Killery AM, et al. Structure and variability of human chromosome ends. Mol Cell Biol 1990; 10: 518-527.

25. Le Bourg E. Correlational analysis in comparative gerontology: An examination of some problems. Exp Gerontol 1996; 31: 645-653.

26. Samani NJ, Boultby R, Butler R, Thompson JR, Goodall AH. Telomere shortening in atherosclerosis. Lancet 2001; 358: 472-473.
27. von Zglinicki T. Role of oxidative stress in telomere length regulation and replicative senescence. Ann NY Acad Sci 2000; 908: 99110.

28. Finkel T, Holbrook NJ. Oxidants, oxidative stress and the biology of ageing. Nature 2000; 408: 239-247.

29. Slagboom PE, Droog S, Boomsma DI. Genetic determination of telomere size in humans: A twin study of three age groups. Am $J$ Hum Genet 1994; 55: 876-882.

30. Elliott WJ, Karrison T. Increased all-cause and cardiac morbidity and mortality associated with the diagonal earlobe crease: A prospective cohort study. Am J Med 1991; 91: 247-254.

31. Kuri M, Hayashi Y, Kagawa K, Takada K, Kamibayashi T, Mashimo $\mathrm{T}$. Evaluation of diagonal earlobe crease as a marker of coronary artery disease: The use of this sign in pre-operative assessment. Anaesthesia 2001; 56: 1160-1162.

32. Pasternac A, Sami M. Predictive value of the ear-crease sign in coronary artery disease. Can Med Assoc J 1982; 126: 645-649.

33. Weber T, Auer J, O'Rourke MF, Kvas E, Lassnig E, Berent R, et al. Arterial stiffness, wave reflections, and the risk of coronary artery disease. Circulation 2004; 109: 184-189.

34. McLeod AL, Uren NG, Wilkinson IB, Webb DJ, Maxwell SR, Northridge DB, et al. Non-invasive measures of pulse wave velocity correlate with coronary arterial plaque load in humans. J Hypertens 2004; 22: 363-368.

35. Koji Y, Tomiyama H, Yamada J, Yambe M, Motobe K, Shiina K, et al. Relationship between arterial stiffness and the risk of coronary artery disease in subjects with and without metabolic syndrome. Hypertens Res 2007; 30: 243-247.

36. Brouilette SW, Moore JS, McMahon AD, Thompson JR, Ford I, Shepherd J, et al. Telomere length, risk of coronary heart disease, and statin treatment in the West of Scotland Primary Prevention Study: A nested case-control study. Lancet 2007; 369: 107-114. 